\title{
Estilos de vida e uso de serviços preventivos de saúde entre adultos filiados ou não a plano privado de saúde (inquérito de saúde de Belo Horizonte)
}

\author{
Lifestyle and preventive health care utilization \\ among adults covered or not by private health plan \\ (the Belo Horizonte health survey)
}

1 Núcleo de Estudos em Saúde Pública e

Envelhecimento da

Fundação Oswaldo Cruz e

Universidade Federal de

Minas Gerais.

Av. Augusto de Lima

1715/60 andar, 30190-002,

Belo Horizonte, M G.

limacosta@cpqrr.fiocruz.br

\begin{abstract}
This study aimed at examining theassociations among private health plan affiliation and health lifestyles, medical counseling about alcohol consumption and tobacco, as well as preventive health care use. The study was carried out in a representative sample of 13,851 residents in the M etropolitan Area of Belo H orizonte (RM BH), aged $>20$ years. From these, $34 \%$ were covered by a private health plan and $66 \%$ were under exclusive public coverage. The former, in comparison with the latter, had healthy lifestyles, regarding current smoking, binge drinking, physical activities during leisure time and consumption of fruits, green and vegetables on daily basis. They had also received more frequent medical advice about smoking and drinking habits. The prevalences of those who received blood pressure and cholesterol assessments, mammography, papanicolau test, as well as fecal occult blood test, were significantly higher among those who were affiliated to a private health plan. All the above mentioned associations were independent of sex, age, and schooling. Our results point to theurgent need of overcoming these inequities.
\end{abstract}

Key words Lifestyles, Preventive health care use, Private health plan, $\mathrm{H}$ ealth survey
Resumo 0 objetivo deste estudo foi investigar as associações entre filiação a plano privado de saúde, alguns estilos de vida, aconsel hamento médico sobre fumo e consumo de álcool euso de serviços preventivos. 0 trabalho foi desenvolvido em uma amostra representativa de residentes na Região M etropolitana de Belo H orizonte com > 20 anos (13.851 participaram). Desses 34\% eram filiados a plano privado e $66 \%$ dependiam exclusivamente do SUS. Os primei ros apresentavam hábitos mais saudáveis: fumavam menos, consumiam menos bebidas alcóolicas, praticavam mais exercícios e consumiam, mais freqüentemente, cinco ou mais porções diárias de frutas, verduras ou legumes frescos. Além disso, haviam recebido, com mais freqüência, aconselhamento médico sobre o consumo de álcool e cigarros. As prevalências de usos de serviços preventivos ( pressão arterial, colesterol, mamografia, exame de papanicolau e pesquisa de sangue oculto nas fezes) eram significativamente mais altas entre os filiados a plano privado de saúde. Todas as associações acima mencionadas eram independentes do sexo, da idade, e da escolaridade. Esses resultados apontam para a premente necessidade de superação dessas iniqüidades.

Palavras-clave Estilos de vida, U so de serviços preventivos, Plano privado de saúde, Inquérito de saúde 


\section{Introdução}

Cerca de um quarto da população adulta brasileira possui cobertura por plano privado de saúde, sendo a filiação a esses planos fundamentalmente determinada pela escolaridade e/ou pela renda familiar (Bahia et al., 2002; LimaCosta et al., 2002). Existem sólidas evidências de que indivíduos filiados a planos privados utilizam serviços de saúde com mais freqüência, em comparação àqueles que dependem exclusivamente do sistema público de saúde. Essas evidências são mais fortes para consultas médicas e mais fracas para internações hospitalares (M cCall et al., 1991; Cohen et al., 1997; Hurd \& M cGarry, 1997; Bahia et al. 2002; Lima-Costa et al., 2002a).

De uma maneira geral, os estudos brasileiros têm mostrado que adultos filiados a plano privado de saúde apresentam melhores condições auto-referidas de saúde (Bahia et al., 2002; Lima-Costa et al., 2002b). Por outro lado, com relação a fatores de riscos cardiovasculares, verificou-se, em um estudo de base populacional conduzido na cidade de Bambuí (MG), que idosos filiados a plano privado de saúde, quando comparados aos que dependiam exclusivamente do Sistema Ú nico de Saúde (SUS), apresentavam medianas semelhantes da pressão sistólica, da pressão diastólica, do colesterol total e do HDL colesterol, assim como de triglicérides (Lima-Costa et al., 2002b). Pelo nosso conhecimento, não existem outros estudos no Brasil sobre o tema.

A Região M etropolitana de Belo Horizonte (RM BH ) é a terceira maior do país em número de habitantes e produção econômica. Recentemente, verificou-se que os adultos residentes na RM BH apresentavam altas prevalências de estilos de vida prejudiciais à saúde: $20 \%$ eram fumantes atuais, $30 \%$ haviam consumido cinco doses de bebidas alcoólicas em uma única ocasião no último mês e somente 3\% haviam comido cinco ou mais porções diárias de frutas, verduras ou legumes frescos nesse período. Além disso, essa população era predominantemente sedentária, pelo menos no que se refere a atividades físicas nos períodos de lazer. Por outro lado, as prevalências de usos de serviços preventivos de saúde eram altas, no que se refere a aferições da pressão arterial e do colesterol, exame de papanicolau e real ização da mamografia (Lima-Costa, 2004a). Via de regra, os estilos de vida prejudiciais à saúde eram mais freqüentes entre adultos com pior escolaridade, observando-se o oposto para o uso de serviços preventivos de saúde, que eram mais freqüente entre aqueles com escolaridade mais alta ( $\mathrm{Li}$ ma-Costa et al., 2004b; 2004c).

0 presente trabalho é parte do inquérito de saúde acima mencionado e tem por objetivo investigar a existência de associação entre filiação a plano privado de saúde e alguns estilos de vida (consumo de álcool e fumo, atividades físicas e consumo de frutas, verduras ou legumes frescos), aconsel hamento profissional sobre fumo e consumo de álcool, além de usos de serviços preventivos de saúde (medidas da pressão arterial, dosagens de colesterol, mamografia, exame de papanicolau e pesquisa de sangue oculto nas fezes). Um objetivo adicional do estudo é verificar a influência da escolaridade nessas associações.

\section{Metodologia}

\section{População estudada}

A RMBH éformada por 24 municípios, com 4,4 milhões de habitantes. 0 presente trabal ho foi conduzido em uma amostra representativa de adultos residentes na referida região metropolitana.

A coleta de dados para a pesquisa foi realizada por meio de um questionário suplementar à Pesquisa de Emprego e Desemprego (PED), conduzida periodicamente na RM BH . Os dados para o inquérito de saúde foram coletados entre 1o de maio e 31 de junho de 2003. A PED/ RM BH é realizada em uma grande amostra, baseada em 7.500 domicílios com cerca de 24.000 moradores. A amostra foi delineada para produzir estimativas da população não institucionalizada, com 10 ou mais anos de idade, residente nos municípios mencionados. Tratase de uma amostra probabilística de conglomerados estratificada em dois estágios. Os setores censitários da Fundação Instituto Brasileiro de Geografia e Estatística (IBGE) representam a unidade primária de seleção e a unidade amostral é o domicílio. As perdas estimadas no cálculo amostral são iguais a 20\%. Dos 7.500 domicílios selecionados, 5.922 (79,0\%) participaram. Para o presente trabalho, foram selecionados todos os participantes da PED/RM BH com idade igual ou superior a 20 anos. A distribuição por sexo e idade dos participantes deste trabal ho e as características correspondentes para a população adulta censitária da 
RMBH foram muito semelhantes. Maiores detalhes podem ser vistos em outras publicações (Fundação João Pinheiro, 1997; LimaCosta, 2004a).

\section{Variáveis do estudo}

A variável dependente deste estudo é a filiação a plano privado de saúde. Essa informação foi obtida por meio da seguinte pergunta: Você tem algum plano privado de saúde?

Três conjuntos de variáveis exploratórias foram considerados: 1) sociodemográficas (sexo, idade e escolaridade); 2) estilos de vida e aconselhamento profissional sobre fumo e consumo de álcool; e 3) uso de serviços preventivos de saúde.

Os seguintes estilos de vida relacionados à saúde foram incluídos no estudo: a) consumo atual de cigarros; b) consumo excessivo de álcool ( 5 ou mais drinques em um único dia nos últimos 30 dias); c) atividades físicas ou exercícios (caminhou para fazer exercícios, ginástica ou esporte por pelo menos 20-30 minutos nos momentos de lazer nos últimos 30 dias); d) autoavaliação do peso (Qual das seguintes frases define seu peso atual?); e) consumo de frutas, verduras e/ou legumes frescos ( $Q$ uantas porções de frutas, verduras ou legumes frescos você consumiu por dia nos últimos 30 dias?). Além desses, foi também considerado aconsel hamento profissional em relação ao cigarro e ao consumo de álcool.

Com relação ao uso de serviços preventivos para doenças e agravos não-transmissíveis, optou-se por considerar a realização desses exames somente nas faixas etárias recomendadas pela Canadian Task Force on Preventive $\mathrm{H}$ ealth Care (Canadian, 2003) e/ou pela U.S. Preventive Services Task Force (US, 2003). Os seguintes exames foram considerados neste trabalho: a) pressão arterial entre homens e mulheres com 20 ou mais anos de idade; b) colesterol entre homens com 35 ou mais anos e mulheres com 45 ou mais anos de idade; c) mamografia entre mulheres com 50-69 anos de idade; d) exame de papanicolau entre mulheres com 20 ou mais anos de idade, que possuíam útero; e) pesquisa de sangue oculto nas fezes entre homens e muIheres com 50 anos ou mais.

0 questionário utilizado para o presente trabalho foi o da pesquisa americana sobre fatores de riscos comportamentais (Behavioral Risk Factor Surveillance), com algumas adaptações (BRFSS, 2001-2002).

\section{Análise dos dados}

A análise dos dados foi realizada utilizando-se os procedimentos do programa STATA (Stata, 2001) para inquéritos populacionais, que incorporam os pesos de cada observação. A análise multivariada foi baseada em odds ratios estimados por meio da regressão logística múltipla (Hosmer \& Lemenshow, 1989). Sexo e idade (variável contínua) foram consideradas a priori variáveis de confusão no estudo.

\section{Resultados}

Dos 13.851 participantes do presente trabalho, 7.457 (53,8\%) eram mulheres e 6.394 $(46,2 \%)$, homens. A média da idade foi igual a 40,5 anos (variação = 20 e 101 anos). A filiação a plano privado de saúde foi relatada por 4.699 $(33,9 \%)$ participantes.

Na tabela 1 está apresentada a distribuição de algumas características sociodemográficas, segundo a filiação a plano privado de saúde. A filiação a plano de saúde foi significativamente mais freqüente entre as mulheres, nas faixas etárias de 30-39, 40-49, 50-59 e 60+ anos de idade, e entre aqueles com escolaridade completa igual a $2 \underline{o}$ grau ou superior. A escolaridade foi a característica sociodemográfica mais fortemente associada à filiação a plano privado de saúde $(O R=2,64$; IC 95\%=2,41-2,90 para 20 grau e OR =9,89; IC 95\% = 8,61-11,37 para curso superior completo).

A distribuição de estilos de vida e de aconselhamento profissional sobre consumo de álcool ou cigarros, segundo a filiação a plano privado de saúde, está apresentada na tabela 2 . A prevalência de fumantes atuais foi menor entre os filiados a plano privado de saúde, mas o consumo excessivo de álcool foi mais freqüente entre eles. Por outro lado, a prevalência de exercícios diários ou quase diários, por 20-30 minutos, durante os períodos de lazer e o consumo diário de $\geq 5$ ou mais porções de frutas, verduras ou legumes frescos foram mais freqüentes entre aqueles que possuíam plano privado de saúde. 0 aconselhamento profissional para ambos, consumo de álcool e de cigarros, foi significativamente mais freqüente entre os filiados a plano privado de saúde. Essas associações persistiram após ajustamentos por idade e sexo.

Todos os usos de serviços preventivos de saúde considerados neste trabal ho foram significativamente mais freqüentes entre aqueles 


\begin{tabular}{|c|c|c|c|}
\hline $\begin{array}{l}\text { Tabela } 1 \\
\text { Distribuição de sexo, } \\
\text { dos habitantes da Regi } \\
\text { a filiação a plano priva }\end{array}$ & $\begin{array}{l}\text { e escolaridad } \\
\text { olitana de Be } \\
\text { le. }\end{array}$ & $\begin{array}{l}\text { nostra de } 13.8 \\
\text { com } 20 \text { ou m }\end{array}$ & $\begin{array}{l}\text { ivíduos representativos } \\
\text { s de idade, segundo }\end{array}$ \\
\hline \multirow[t]{2}{*}{ Características } & \multicolumn{2}{|c|}{ Filiação a plano privado de saúde \% } & \multirow[t]{2}{*}{ Odds ratio (IC 95\%) } \\
\hline & Sim & Não & \\
\hline \multicolumn{4}{|l|}{ Sexo } \\
\hline M asculino & 44,7 & 47,0 & 1,00 \\
\hline Feminino & 55,3 & 53,0 & $1,10(1,01-1,19)$ \\
\hline \multicolumn{4}{|l|}{ Faixa etária } \\
\hline $20-29$ & 25,3 & 33,7 & 1,00 \\
\hline $30-39$ & 23,0 & 23,2 & $1,32(1,18-1,48)$ \\
\hline $40-49$ & 19,2 & 21,5 & $1,49(1,32-1,68)$ \\
\hline $50-59$ & 15,5 & 13,3 & $1,55(1,36-1,77)$ \\
\hline$>60$ & 14,7 & 10,6 & $1,85(1,62-2,11)$ \\
\hline \multicolumn{4}{|l|}{ Escolaridade completa } \\
\hline M enos que 20 grau & 34,6 & 67,5 & 1,00 \\
\hline 20 grau & 36,3 & 26,8 & $2,64(2,41-2,90)$ \\
\hline Superior & 29,1 & 5,7 & $9,89(8,61-11,37)$ \\
\hline
\end{tabular}

OR (IC 95\%): Odds ratio bruto e intervalo de confiança em nível de 95\%.

que possuíam plano privado de saúde, mesmo após ajustamentos por sexo e idade (Tabela 3).

$\mathrm{Na}$ tabela 4 está apresentada a distribuição de estilos de vida e de aconsel hamento médico sobre consumo de álcool e cigarros entre aqueles que possuíam ou não filiação a plano privado de saúde, segundo a escolaridade ( $2 \underline{\text { o grau }}$ completo ou menos). 0 hábito de fumar foi significativamente menos freqüente entre os filiados a plano privado de saúde, tanto entre aqueles que possuíam segundo grau completo, quanto entre os que apresentavam menor escolaridade. Da mesma forma, a realização de exercícios diários ou quase diários nos momentos de lazer, a ingestão diária de cinco ou mais porções de frutas, verduras ou legumes frescos, assim como 0 aconsel hamento profissional sobre consumo de álcool ou cigarros, foram mais freqüentes entre os filiados a plano privado de saúde, em ambos os estratos de escolaridade. Por outro lado, após estratificação por escolaridade, não se observou associação significante entre o consumo excessivo de bebidas alcoólicas e filiação a plano privado de saúde.

Como pode ser observado na tabela 5, entre aqueles com menor escolaridade, todos os usos de serviços preventivos de saúde apresentaram associações significantes, e independentes da idade e sexo, com filiação a plano privado de saúde. Entre os que possuíam 2o grau completo, as associações correspondentes foram en- contradas para todos os exames considerados, exceto pesquisa de sangue oculto nas fezes.

\section{Discussão}

A escolaridade foi a característica sociodemográfica mais fortemente associada à filiação a um plano privado de saúde entre adultos participantes do inquérito de saúde da RM BH , confirmando pesquisas brasileiras anteriores, utilizando a base de dados da Pesquisa Nacional por Amostras de Domicílio (Bahia et al., 2002; Lima-Costa et al., 2002).

No presente trabalho, de uma maneira geral, aqueles filiados a plano privado de saúde apresentavam hábitos mais saudáveis. Eles fumavam menos, consumiam menos bebidas alcoólicas, praticavam mais exercícios nos períodos de lazer e comiam com mais freqüência cinco ou mais porções de frutas, verduras ou legumes frescos em bases diárias. Além disso, com mais freqüência, eles haviam recebido aconselhamento médico sobre o consumo de álcool ou cigarros. A associação entre filiação a plano privado de saúde e hábitos mais saudáveis (exceto consumo de álcool) foi observada tanto entre aqueles que possuíam 2 o grau completo, quanto entre aqueles com escolaridade inferior. 0 delineamento seccional dessa pesquisa não permite estabelecer relação temporal. Des- 
Tabela 2

Distribuição de estilos de vida relacionados à saúde e de aconsel hamento médico sobre consumo de álcool ou cigarros em uma amostra de 13.851 indivíduos representativos dos habitantes da Região M etropolitana de Belo H orizonte com 20 ou mais anos de idade, segundo a filiação a plano privado de saúde.

\begin{tabular}{|c|c|c|c|}
\hline \multirow[t]{2}{*}{ Características } & \multicolumn{2}{|c|}{$\begin{array}{l}\text { Filiação a plano privado } \\
\text { de saúde } \%\end{array}$} & \multirow[t]{2}{*}{$\begin{array}{l}\text { Odds ratio reajustado } \\
\text { por sexo e idade (IC } 95 \%\end{array}$} \\
\hline & Sim & Não & \\
\hline Fuma atualmente & 15,2 & 23,9 & $0,61(0,55-0,67)$ \\
\hline $\begin{array}{l}\text { Ingestão de } \geq 5 \text { doses de bebidas } \\
\text { alcoólicas no último mês em um único dia }\end{array}$ & 31,6 & 28,3 & $1,28(1,16-1,40)$ \\
\hline $\begin{array}{l}\text { Exercícios diários, ou quase, nos momentos de lazer } \\
\text { por pelo menos 20-30 minutos nos últimos } 90 \text { dias }\end{array}$ & 14,5 & 9,2 & $1,62(1,43-1,84)$ \\
\hline Avalia seu peso como acima do desejado & 57,6 & 42,4 & $1,34(1,22-1,47)$ \\
\hline $\begin{array}{l}\text { Consumo diário de } \geq 5 \text { porções de frutas, } \\
\text { verduras ou legumes frescos nos últimos } 30 \text { dias }\end{array}$ & 5,4 & 2,0 & $2,66(2,12-3,34)$ \\
\hline $\begin{array}{l}\text { Alguma vez na vida recebeu aconselhamento } \\
\text { de médicos ou profissionais de saúde sobre } \\
\text { cigarros (somente os que fumam atualmente) }\end{array}$ & 75,9 & 59,8 & $2,02(1,61-2,54)$ \\
\hline $\begin{array}{l}\text { Alguma vez na vida recebeu aconselhamento } \\
\text { de médicos ou profissionais de saúde sobre } \\
\text { consumo de álcool (somente bebedores excessivos) }\end{array}$ & 67,4 & 49,1 & $2,04(1,74-2,39)$ \\
\hline
\end{tabular}

OR (IC 95\%): Odds ratio e intervalo de confiança em nível de $95 \%$, ajustado por sexo e idade.

$\%$ : percentagem dos que disseram sim.

\section{Tabela 3}

Distribuição dos usos de al guns serviços preventivos de saúde em uma amostra de 13.851 indivíduos representativos dos habitantes da Região M etropolitana de Belo Horizonte com 20 ou mais anos de idade, segundo a filiação a plano privado de saúde.

\begin{tabular}{llcl}
\hline Características & $\begin{array}{c}\text { Filiação a plano privado } \\
\text { de saúde } \%\end{array}$ & Odds ratio (IC 95\%) \\
\hline & Sim & Não & \\
\hline $\begin{array}{l}\text { D eterminação da pressão arterial há } \leq 2 \text { anos } \\
\text { (homens e mulheres com } \geq 20 \text { anos de idade) }\end{array}$ & 96,3 & 88,7 & $3,05(2,54-3,67)$ \\
\hline $\begin{array}{l}\text { Dosagem de colesterol há } \leq 5 \text { anos (homens com } \geq \\
35 \text { anos de idade e mulheres com } \geq 45 \text { anos de idade) }\end{array}$ & 96,9 & 87,9 & $2,40(1,97-2,93)$ \\
\hline $\begin{array}{l}\text { Pesquisa de sangue nas fezes há } \leq 2 \text { anos } \\
\text { (homens e mulheres com } \geq 50 \text { anos de idade) }\end{array}$ & 21,8 & 15,4 & $1,52(1,24-1,86)$ \\
\hline $\begin{array}{l}\text { M amografia há } \leq 2 \text { anos (mulheres com 50-69 anos) } \\
85,6\end{array}$ & 65,3 & $3,35(2,51-4,48)$ \\
\hline $\begin{array}{l}\text { Exame de papanicolau há } \leq 3 \text { anos (mulheres com } \\
\geq 20 \text { anos de idade que possuem útero) }\end{array}$ & 85,6 & 71,6 & $2,36(2,04-2,73)$ \\
\hline $\begin{array}{l}\text { OR (IC 95\%): Odds ratio e intervalo de confiança em nível de 95\%, ajustado por sexo e idade. } \\
\%: \text { percentagem dos que disseram sim. }\end{array}$
\end{tabular}


Tabela 4

Distribuição de estilos de vida relacionados à saúde e de aconsel hamento médico para hábitos selecionados em uma amostra de 13.851 indivíduos representativos dos habitantes da Região M etropolitana de Belo Horizonte com 20 ou mais anos de idade, segundo a filiação a plano privado de saúde e escolaridade.

\begin{tabular}{|c|c|c|c|c|c|c|}
\hline \multirow[t]{3}{*}{ Características } & \multicolumn{3}{|c|}{20 grau completo } & \multicolumn{3}{|c|}{ Menos que 20 grau completo } \\
\hline & \multicolumn{2}{|c|}{ Plano Privado } & \multirow[t]{2}{*}{ OR (IC 95\%) } & \multicolumn{2}{|c|}{ Plano Privado } & \multirow[t]{2}{*}{ OR (IC 95\%) } \\
\hline & Sim & Não & & Sim & Não & \\
\hline Fuma atualmente & 14,3 & 15,8 & $0,81(0,67-0,99)$ & 17,0 & 26,5 & $0,64(0,55-0,75)$ \\
\hline $\begin{array}{l}\text { Ingestão de } \geq 5 \text { doses de bebidas alcoólicas } \\
\text { no último mês em um único dia }\end{array}$ & 35,4 & 30,2 & $1,14(0,97-1,34)$ & 24,6 & 27,4 & $1,06(0,91-1,23)$ \\
\hline $\begin{array}{l}\text { Exercícios diários, ou quase, nos momentos de lazer } \\
\text { por pelo menos 20-30 minutos nos últimos } 90 \text { dias }\end{array}$ & 16,1 & 10,8 & $1,54(1,23-1,93)$ & 11,4 & 8,5 & $1,31(1,09-1,59)$ \\
\hline $\begin{array}{l}\text { Consumo diário de } \geq 5 \text { porções de frutas, verduras } \\
\text { ou legumes frescos nos últimos } 30 \text { dias }\end{array}$ & 6,2 & 2,7 & $1,82(1,24-2,69)$ & 3,6 & 1,7 & $1,96(1,40-2,75)$ \\
\hline $\begin{array}{l}\text { Alguma vez na vida recebeu aconsel hamento } \\
\text { de médicos ou profissionais de saúde sobre } \\
\text { cigarros ( somente os que fumam atualmente) }\end{array}$ & 75,9 & 65,3 & $1,63(1,09-2,44)$ & 75,9 & 58,2 & $2,14(1,55-2,96)$ \\
\hline $\begin{array}{l}\text { Alguma vez na vida recebeu aconselhamento de } \\
\text { médicos ou profissionais de saúde sobre consumo } \\
\text { de álcool (somente bebedores excessivos) }\end{array}$ & 68,7 & 52,9 & $1,57(1,10-2,06)$ & 63,9 & 47,0 & $1,81(1,40-2,33)$ \\
\hline
\end{tabular}

OR (IC 95\%): Odds ratio e intervalo de confiança em nível de 95\%, ajustado por sexo e idade.

\section{Tabela 5}

Distribuição dos usos de serviços preventivos de saúde em uma amostra de 13.851 indivíduos representativos dos habitantes da Região M etropolitana de Belo Horizonte com 20 ou mais anos de idade, segundo a filiação a plano privado de saúde e escolaridade.

\begin{tabular}{|c|c|c|c|c|c|c|}
\hline \multirow[t]{3}{*}{ Características } & \multicolumn{3}{|c|}{20 grau completo } & \multicolumn{3}{|c|}{ Menos que 20 grau completo } \\
\hline & \multicolumn{2}{|c|}{ Plano Privado } & \multirow[t]{2}{*}{ OR (IC 95\%) } & \multicolumn{2}{|c|}{ Plano Privado } & \multirow[t]{2}{*}{ OR (IC 95\%) } \\
\hline & Sim & Não & & Sim & Não & \\
\hline $\begin{array}{l}\text { Determinação da pressão arterial há } \leq 2 \text { anos } \\
\text { (homens e mulheres com } \geq 20 \text { anos de idade) }\end{array}$ & 96,0 & 88,6 & $2,37(1,84-3,06)$ & 96,9 & 88,6 & $3,14(2,34-4,21)$ \\
\hline $\begin{array}{l}\text { Dosagem de colesterol há } \leq 5 \text { anos (homens com } \geq \\
35 \text { anos de idade e mulheres com } \geq 45 \text { anos de idade) }\end{array}$ & 97,2 & 90,2 & $1,63(1,26-2,10)$ & 96,0 & 87,4 & $2,35(1,78-3,11)$ \\
\hline $\begin{array}{l}\text { Pesquisa de sangue nas fezes há } \leq 2 \text { anos (homens } \\
\text { e mulheres com } \geq 50 \text { anos de idade) }\end{array}$ & 25,6 & 21,6 & $1,17(0,77-1,78)$ & 18,8 & 14,5 & $1,32(1,02-1,70)$ \\
\hline Mamografia há $\leq 2$ anos (mulheres com 50-69 anos) & 92,3 & 73,6 & $5,00(2,56-9,76)$ & 79,9 & 64,0 & $2,47(1,75-3,48)$ \\
\hline $\begin{array}{l}\text { Exame de papanicolau há } \leq 3 \text { anos (mulheres com } \\
\geq 20 \text { anos de idade que possuem útero) }\end{array}$ & 87,8 & 66,6 & $2,70(2,17-3,36)$ & 82,4 & 74,8 & $1,84(1,47-2,30)$ \\
\hline
\end{tabular}

$\geq 20$ anos de idade que possuem útero)

OR (IC 95\%): Odds ratio e intervalo de confiança em nível de 95\%, ajustado por sexo e idade. 
sa forma, não é possível saber: 1) se a influência da filiação a um plano privado de saúde leva a menores exposições de risco; ou 2) se indivíduos mais preocupados com a própria saúde procuram com mais freqüência a cobertura por esses planos. M as é importante salientar que os planos privados de saúde da RM BH não possuem atividades regulares de promoção da saúde. Dessa forma, é razoável supor que a segunda hipótese seja mais plausível.

As principais causas de mortalidade entre adultos brasileiros são as doenças do aparelho circulatório e as neoplasias. Entre as primeiras, predominam as doenças cerebrovasculares e as doenças isquêmicas do coração. Entre as últimas, predominam as neoplasias malignas da traquéia, brônquios e pulmões entre os homens, seguidas pela da próstata, do estômago, do esôfago e do cólon, reto ou ânus. A neoplasia maligna mais freqüente como causa de mortalidade entre brasileiras adultas éa da mama, seguida pela da traquéia, brônquios e pulmões, do cólon, reto ou ânus, do colo do útero e do estômago (M inistério da Saúde, 1996-2000). Existem evidências de que a detecção de casos, e conseqüente tratamento, pode reduzir a mortalidade por várias das causas de mortalidade acima mencionadas. Entretanto, essas evidências muitas vezes são restritas a certas faixas etárias e a intervalos predeterminados entre os exames. Duas forças-tarefa, uma no Canadá (CTFPHC, 2003) e outra nos Estados Unidos (USPSTF, 2003), estabeleceram consensos abrangentes sobre este tema. De uma maneira geral, recomenda-se: 1) determinação da pressão arterial a cada dois anos entre homens e mulheres com 20 ou mais anos de idade; 2) dosagem de colesterol a cada cinco anos entre homens com 35 ou mais anos e mulheres com 45 ou mais anos de idade; 3) exame de papanicolau a cada três anos entre mulheres com 18-20 anos de idade; e 4) pesquisa de sangue oculto nas fezes a cada dois anos entre homens e mulheres com 50 ou mais anos de idade (CTFPHC, 2003; USPSTF, 2003). Com relação à mamografia, existe al guma controvérsia. 0 grupo americano recomenda a realização rotineira da mesma em mulheres com idade igual ou superior a 40 anos (USPSTF, 2003), ao passo que o grupo canadense recomenda a sua utilização, como parte do exame rotineiro de saúde, somente na faixa etária de 50-69 anos, uma vez que as evidências da efetividade desse exame para a redução da mortalidade por câncer de mama são mais fortes nessa faixa etária (CTFPHC, 2003).
No presente trabalho, todos os serviços pre ventivos de saúde, nas faixas etárias e freqüências acima recomendadas, foram significativamente mais freqüentes entre os filiados a plano privado de saúde, independentemente do sexo e da escolaridade. Os Centers for Disease Control and Prevention, por meio do documento $\mathrm{H}$ ealthy People 2010, estabeleceram algumas metas, para realização de exames preventivos, a serem alcançadas nos Estados U nidos no final da presente década (CDC, 2003). Este documento estabelece, como metas: 1) determinação da pressão arterial a cada dois anos em $95 \%$ da população com $\geq 20$ anos de idade; 2 ) aferição da colesterolemia a cada cinco anos em $80 \%$ da população com $\geq 20$ anos de idade; 3) realização bianual da mamografia em $70 \%$ das mulheres com $\geq 40$ anos de idade; 4) realização trianual do exame de papanicolau em $90 \%$ daquelas com $\geq 20$ anos de idade que possuem útero; e 5) realização bianual da pesquisa de sangue oculto nas fezes a partir dos 50 anos de idade (CDC, 2003). Na presente investigação, verifica-se que essas metas foram alcançadas, entre os filiados a plano privado de saúde, para as determinações da pressão arterial (96\%) e da mamografia ( $91 \%$ na faixa etária $\geq$ 40 anos; dados não apresentados ), mas não entre os SU S dependentes ( $89 \%$ e $61 \%$, respectivamente). Com relação às aferições da colesterolemia, as metas foram praticamente alcançadas entre filiados e não filiados a plano privado ( $82 \%$ e $79 \%$ na faixa $\geq 20$ anos, respectivamente; dados não apresentados). 0 exame de papanicolau na faixa etária igual a > 20 anos esteve abaixo da meta estabelecida, em ambos os grupos ( 86 e $72 \%$, respectivamente). Cabe destacar a baixíssima prevalência da realização da pesquisa de sangue oculto nas fezes, tanto entre os filiados a plano privado de saúde (22\%), quanto entre os SUS dependentes (15\%).

Um resultado intrigante deste trabal ho foi a persistência da associação entre plano privado de saúde e uso de todos os serviços preventivos considerados (exceto pesquisa de sangue nas fezes), após estratificação por escolaridade. São necessárias investigações mais profundas para uma melhor compreensão dessas associações.

Em resumo, nossos resultados mostraram que existiam diferenças de estilos de vida entre adultos filiados ou não a plano privado de saúde, observando-se hábitos mais saudáveis entre os primeiros, independente do nível de escolaridade. A prevalência de uso de serviços preventivos neste trabal ho foi, também, significativa- 
mente mais alta entre os filiados a plano privado, em comparação àqueles que dependiam exclusivamente do SUS, mesmo estratificandose esses resultados pela escolaridade. Esses resultados apontam para a premente necessi dade de superação das iniqüidades constatadas.

\section{Agradecimentos}

Este trabalho foi desenvolvido como parte das atividades do Núcleo de Estudos em Saúde Pública e Envelhecimento da Fundação Oswaldo Cruz e Universidade Federal de M inas Gerais (NESPE/Fiocruz-UFM G), como Centro Colaborador da Secretaria de Vigilância à Saúde do M inistério da Saúde (SVS-MS) em Saúde do Idoso. 0 trabaIho foi financiado pela SVS-M S e pelo Banco Mundial.

\section{Referências bibliográficas}

Bahia L, Costa AJL, Fernandes C, Luiz RR \& Cavalcanti M LT 2002. Segmentação da demanda dos planos e seguros privados de saúde: uma análise das informações da PNAD/98. Ciência e Saúde Coletiva 7: 671-686.

Canadian Task Force on Preventive Health Care Force [acessado no ano de 2003 para informações relativas a 2003]. Disponível em <http:www.ctfphc.org>

Centers for Disease Control and Prevention. Behavioral Risk Factor Surveillance: BRFSS [acessados durante os anos de 2001 e 2002 para informações relativas a 2001 e 2002]. Disponível em <http:www.cdc.gov/bbrfss>

Centers for Disease Control and Prevention. Department of $\mathrm{H}$ ealth and Human Services. Healthy People 2010. Review, 2003. <http://www.healthypeople.gov>

Cohen RA, Bloom B, Simpsom G \& Pearsons E 1997. ACcess to health care. Part 3: Older adults. Vital and $\mathrm{H}$ ealth Statistics. Series 10. Data from the National Health Survey, 1998:1-32.

Fundação João Pinheiro 1997. Pesquisa de Emprego e De semprego na Região M etropolitana de Belo H orizonte (PED/RM BH ): Plano de Trabalho - SINE M G, Belo Horizonte: Secretaria do Planejamento do Estado de Minas Gerais.

Hosmer DW \& Lemenshow S 1989. Applied logistic regression. Johns Wiley and Sons, Nova York.

Hurd M D \& M cGarry K 1997. Medical insurance and the use of health care services by the elderly. Journal of Health Economics 16:129-154.

Lima-Costa M F, Barreto S, Giatti L 2002a. A situação socioeconômica afeta igualmente a saúde de idosos e adultos mais jovens no Brasil? Um estudo utilizando dados da Pesquisa Nacional por Amostras de Domicílios - PNAD/98. Ciência eSaúdeColetiva 7:813-824.
Lima-Costa M F et al. 2002b. The Bambuí Health and Ageing Study (BHAS): private health plan and medical care utilization by older adults. Cadernos de Saúde Pública 18:177-186.

Lima-Costa M F 2004a. A saúde dos adultos na Região Me tropolitana de Belo Horizonte: um estudo epidemiológico de base populacional. Belo Horizonte: $\mathrm{Núcleo}$ de Estudos em Saúde Pública e Envelhecimento. $<$ <ww.cpqrr.fiocruz.br/NESPE>

Lima-Costa M F 2004b. A escolaridade afeta igualmente comportamentos prejudiciais à saúde de idosos e adultos mais jovens? (Inquérito de Saúde da Região Metropolitana de Belo Horizonte). Epidemiologia e Serviços de Saúde. (No prelo).

Lima-Costa M F 2004C. Influência da idade e da escolaridade no uso de serviços preventivos de saúde. (Inquérito de Saúde da Região M etropolitana de Belo Horizonte). Epidemiologia e Serviços de Saúde. (No prelo).

McCall NT, Rice J, Boismier J \& West R 1991. Private health insurance and medical care utililization: evidence from the M edicare population. Inquirity 28 : 276-287.

M inistério da Saúde. Secretaria de Informática. Departamento de Informática do Sistema Ú nico de Saúde. Sistema de Informações sobre M ortalidade (SIM ), 1996-2000 (CD Rom)

Stata Statistical Software (computer program) Release 7.0. Texas: College Stations, Stata Corporation; 2001.

U.S. Preventive Services Task Force [acessado no ano de 2003 para informações relativas a 2003]. Disponível em <http:www.ahcpr.org/>

Artigo apresentado em 20/4/2004

Aprovado em 10/5/2004

Versão final apresentada em 20/5/2004 